\title{
Emociones y aprendizaje informal en Biología ¿Qué lugar le damos en la formación del profesorado?
}

\section{Emoções e aprendizagem informal em Biologia. Que lugar damos a ela na formação de professores?}

\author{
Arabela Beatriz Vaja (avaja@unvm.edu.ar) \\ Universidad Nacional de Villa María y Universidad Nacional de Río Cuarto, Córdoba, Argentina. \\ Nahuel Ezequiel Palombo (npalombo@imbiv.unc.edu.ar) \\ Instituto Multidisciplinario de Biología Vegetal (IMBIV), CONICET-UNC, Córdoba, Argentina.
}

Rocío Belén Martín (rbmartin@unc.edu.ar)

Facultad de Ciencias Exactas, Físicas y Naturales, UNC - Centro de Investigaciones y Transferencia Villa María CONICET, Córdoba, Argentina.

Resumen: El aprendizaje es un proceso que dura toda la vida y en el que las personas co-construyen conocimientos, habilidades socio emocionales y actitudes mediante las experiencias cotidianas y su relación con el ambiente. En los últimos años, surgieron estudios orientados al aprendizaje informal en Ciencias y a los aspectos emocionales y afectivos implicados en los contextos educativos. En este trabajo se describen y analizan los relatos e interacciones surgidas en una tarea propuesta en el marco de un curso de formación docente virtual, en que se solicitaba a sus participantes pensar cómo aprenden Biología en su vida cotidiana. Los resultados mostraron que los profesores expresan sus vivencias y emociones en cuanto a los aprendizajes cotidianos; brindando una oportunidad para que reflexionen sobre sus roles en educación, haciendo explícito sus formas de aprender y conocer cómo los estudiantes aprenden en contextos cotidianos. Se reflexiona sobre la formación docente: por un lado, acerca de la importancia de incluir propuestas y temas que promuevan emociones beneficiosas para los aprendizajes; y por otro lado, sobre la relevancia que tiene repensar los contextos de aprendizajes y conocer otros ámbitos en los cuales pueden intervenir y fomentar el desarrollo de actitudes acordes a estos nuevos desafíos.

Palabras clave: Saberes cotidianos; Aspectos afectivos; Docentes; Aprendizaje permanente; Ciencias Naturales.

Resumo: A aprendizagem é um processo ao longo da vida em que as pessoas coconstroem conhecimentos, habilidades e atitudes socioemocionais por meio de experiências cotidianas e de sua relação com o meio ambiente. Nos últimos anos, tem havido estudos voltados para a aprendizagem informal em ciências e os aspectos emocionais e afetivos envolvidos em contextos educacionais. Neste artigo, descrevemos e analisamos as histórias e interações que surgiram em um curso proposto no âmbito de um curso virtual de formação de professores, no qual os participantes foram convidados a refletir sobre como aprenderam Biologia em seu cotidiano. Os resultados mostram que os professores expressam suas experiências e emoções a partir do aprendizado diário; 
dando-lhes a oportunidade de refletir sobre seus papéis na educação, explicitando suas formas de aprendizagem e sabendo como os alunos aprendem em contextos cotidianos. Reflexão sobre a formação de professores: por um lado, sobre a importância de incluir propostas e temas que promovam emoções benéficas para a aprendizagem; e, por outro lado, na relevância que necessita para repensar os contextos de aprendizagem e conhecer outras áreas onde possam intervir e promover o desenvolvimento de atitudes de acordo com estes novos desafios.

Palavras-chave: Conhecimento cotidiano; Aspectos afetivos; Professores; aprendizagem permanente; Ciências Naturais

\section{INTRODUCCIÓN}

Por lo tanto, mi éxito como hombre de ciencia, cualquiera que sea la altura que haya alcanzado, ha sido determinado, en la medida que puedo juzgar, por complejas y diversas cualidades y condiciones mentales. De ellas, las más importantes han sido: a) la pasión por la ciencia; b) paciencia ilimitada para reflexionar largamente sobre cualquier tema; c) laboriosidad en la observación y recolección de datos y d) una mediana dosis de inventiva así como de sentido común. (DARWIN, s/f, 71).

La observación, la paciencia... y la pasión son expresividades y cualidades que se despliegan ciertamente y en general en contextos y situaciones de la cotidianeidad. ¿Aprendemos siempre? ¿Dejamos de aprender en algún momento? ¿Resulta imposible no aprender del ambiente? ¿Qué sentimos frente a esos aprendizajes cotidianos? ¿Aprendemos sobre empatía verde? El aprendizaje es un proceso que dura toda la vida y en el que las personas co-construyen conocimientos, habilidades socio emocionales y actitudes mediante las experiencias cotidianas y su relación con el ambiente.

En este trabajo se describen y analizan los comentarios e interacciones en una tarea de un curso de formación docente virtual, que consideró la inclusión de nuevos temas como las emociones y aprendizajes informales cotidianos en Biología.

En un primer y segundo apartado se presentarán los referentes teóricos de este trabajo, por un lado aquellos referidos a los aprendizajes informales en biología y por otro, a las emociones en el profesorado. En un tercer apartado se hará alusión al método de estudio, para proseguir con los resultados derivados de los relatos de los profesores. Para finalizar, se expondrán las consideraciones finales del estudio.

\section{APRENDIZAJES INFORMALES EN BIOLOGÍA}


En el campo de la educación en ciencias, se evidencia una literatura escasa en relación con los aprendizajes informales. Estudios recientes reconocen que hay variados contextos para el aprendizaje: zoológicos, acuarios, museos, jardines botánicos, huertas, viveros, revistas, películas, documentales de televisión, Internet, el aprendizaje en el campo, a través de biografías, entre otros, que son de interés y deberían ser estudiados (PALOMBO, 2020; REISS y McCOMAS, 2020).

En este sentido, el aprendizaje informal es entendido como el que acontece indiferenciada y subordinadamente a otros procesos sociales; en sí, cuando se está inmerso en otras realidades culturales (TRILLA, 1992 en MARTÍN y DONOLO, 2019). Es resultante de las actividades de la vida diaria relacionadas con el trabajo, la familia o el ocio, conociéndose a menudo como aprendizaje experimental o accidental, precisamente porque no se encuentra estructurado en términos de objetivos de aprendizaje, tiempo de aprendizaje o apoyo de aprendizaje (FORESTO, 2020).

Creemos que pensar el aprendizaje informal no puede dejar de asociarse a las ideas de cognición y aprendizaje situado, que se fueron produciendo tras descubrir y focalizar en los aspectos de la cognición cotidiana esenciales para todo aprendizaje, tales como la resolución de dificultades emergentes, la utilización de los planes como recursos y limitaciones de la acción situada, y la consideración de los significados como objeto de negociación social en contextos concretos (STREIBEL, 1993 en MARTÍN, 2019).

Dentro de las incipientes contribuciones al estudio del aprendizaje informal en ciencias, puede reconocerse que el término se ha utilizado con dos significados diferentes o en al menos dos áreas de estudio distintas pero que se imbrican. Algunos usan la frase para considerar al aprendizaje que ocurre en todos los tipos de entornos informales de educación científica, como los entornos públicos, no escolares, diseñados como los museos de ciencias y los clubes extracurriculares, los hogares, patios de recreo, con los compañeros y en otras situaciones en las que hay una agenda educativa explícitamente diseñada y planificada (FALK, 2006; RENNIE, 2014; KIM; DOPICO, 2016). Otros excluyen a los entornos diseñados y se refieren sólo a los entornos no diseñados, sin una intencionalidad educativa detrás (ESHACH, 2007; MIYAKE, 2017). A partir de estas consideraciones, es posible advertir que la definición de aprendizaje informal está sesgada por aquellos esfuerzos por diferenciar o comparar el aprendizaje 
Edição Especial: I SSAPEC - Simpósio Sul-Americano de Pesquisa em Ensino de Ciências ISSN: 2595- $4520 \quad$ Vol. 4, n. 3. 2021

informal y formal, y estas opiniones dicotomizadas con frecuencia son simplificaciones excesivas de las características del aprendizaje informal. Sin embargo, la naturaleza del aprendizaje en ambientes informales es mucho más compleja. Debido a que tal aprendizaje es complejo, el intento de dicotomizar es un intento de reducir la complejidad inherente a la naturaleza intrincada del aprendizaje y la diversidad de puntos de vista y valores (ANDERSON; ELLENBOGEN, 2012).

En concordancia, en una distinción de los aprendizajes informales, Reiss y McComas (2020) exponen que el aprendizaje extraescolar se divide, por un lado, en aquellos "sitios formales-informales", con objetivos explícitos de aprendizaje relativos a la programación educativa. Y por otro lado, los sitios "informales-informales", donde el aprendizaje probablemente es reconocido pero no tiene un objetivo o intención educativa por detrás.

El entendimiento y el abordaje del aprendizaje informal en ciencias biológicas no puede separarse de los planteos existentes en cuanto a la importancia de la coconstrucción de identidades ambientales y de narrativas y relatos de la naturaleza; al respecto Bruner (2003) considera que la identidad deriva en su mayoría de las historias que nos vamos contando a nosotros mismos, enlazando los diferentes fragmentos y partes que componen nuestra vida, la co-construcción de la identidad, no puede avanzar sin la capacidad y posibilidad de narrar. La cultura forma parte de nosotros y aporta herramientas para co-construir nuestro propio mundo, como también nuestras concepciones acerca de nosotros mismos; por ello, la educación y el aprendizaje deben entenderse necesariamente en ese contexto cultural en que se insertan, ya que toda actividad mental está 'culturalmente situada', y por lo tanto, no puede entenderse fuera de ese contexto que le da la posibilidad de existir, dándole forma y amplitud (VAJA, 2014).

En este sentido, emergen planteos que destacan la relevancia de considerar otras formas de aprender, atendiendo a las emociones y a las habilidades socio emocionales en relación a los contextos culturales, la identidad ambiental y los saberes cotidianos.

\section{EMOCIONES EN EL PROFESORADO}


El estudio de aspectos emocionales fue cobrando importancia en los últimos años, desde el ámbito de la Psicología Educacional. Así, muchos trabajos comenzaron a reconocer estos aspectos como importantes dentro de los contextos educativos y un ejemplo de ello es la teoría de Pekrun (2006). Si bien desde la teoría hay una amplia producción en torno a las emociones de los estudiantes, no se desconocen las emociones de los docentes.

Así, comienzan a estudiarse y surge una diversidad de trabajos en los cuales las emociones son abordadas en relación con otros importantes aspectos considerados por la Psicología educacional. Fried, Mansfield y Dobozy (2015) realizaron una revisión de diferentes publicaciones en las que se abordaron las emociones de los docentes. A partir de esa revisión, señalan que se fueron estableciendo relaciones entre éstas y otros aspectos de la vida en el aula. De este modo, las emociones se vincularon con las relaciones entre docentes y estudiantes, con el ambiente de aprendizaje y el compromiso de los estudiantes, con las emociones de los estudiantes y con la habilidad de los docentes de manejar los cambios y la incertidumbre. Según los autores mencionados, todos estos estudios destacan la importancia de las emociones de los docentes y el potencial que dichas emociones tienen para influir en la vida del aula.

Una cuestión que resulta interesante considerar al respecto, es la idea de 'contagio emocional'. Según Pekrun (2014) las emociones son contagiosas y pueden transmitirse, por lo tanto las emociones que un docente experimenta y manifiesta, puede llegar a tener efectos en las emociones de los estudiantes. Por este motivo, el autor mencionado advierte que el docente debe prestar atención acerca de sus emociones: sería conveniente que muestre emociones positivas sobre la enseñanza y sobre lo que enseña de modo que comparta con los estudiantes emociones como disfrute, alegría y entusiasmo.

A partir de los planteos expuestos hasta aquí en relación con los avances actuales sobre aprendizajes informales y las emociones en el profesorado, nos preguntamos ¿qué actividades cotidianas nos sirven para aprender y disfrutar de los aprendizajes construidos? ¿Son reconocidas y valoradas por los docentes como actividades promotoras de disfrute y alegría? ¿Cómo contribuir a que identifiquen las propias emociones positivas para que luego promuevan el entusiasmo y la pasión por la ciencia en sus estudiantes? ¿Qué tipos de espacios y actividades podemos generar -en el marco 
de procesos de formación continua- para que los docentes puedan reflexionar acerca de los aspectos afectivos que se ponen en juego al momento de enseñar, tanto dentro de la escuela como fuera de ella?

\section{METODOLOGÍA}

El estudio de caso refiere a un curso de formación docente sobre emociones y compromiso en diversos contextos -formales, no formales e informales-, para profesores y adscriptos de diferentes carreras en Ciencias y Tecnología (Biología, Geología e Ingenierías) de una universidad pública de Córdoba, Argentina. Durante los meses de mayo y junio del año 2020 se desarrolló un curso de 30h de duración, con modalidad gratuita y abierta a la comunidad académica de la facultad. Contó con la participación de 17 personas, todas ellas interesadas en la docencia, cinco se desempeñan en el área de las ciencias biológicas, ocho en el campo de las ingenierías (biomédica, química, etc.), tres en el área de geología y una persona encargada de la parte de bibliotecología.

Se desarrollaron cinco módulos virtuales que abordaron diversas temáticas, a saber: (1) contextos y Ecologías de aprendizaje, (2) emociones en los aprendizajes, (3) compromiso en los aprendizajes, (4) aprendizaje de la Biología e Ingeniería en contextos diversos y (5) una integración final.

Durante el desarrollo del curso se realizaban actividades en todos los encuentros virtuales, que implicaron exposiciones combinadas con estrategias como análisis de vídeos, cuestionarios, lecturas de biografías y artículos científicos, reflexiones sobre producciones expresivas y culturales, elaboración de relatos en cuanto a ciertos temas vinculados a los aprendizajes y una evaluación final que implicaba una autobiografía de aprendizaje, que atendía a emociones y a la diversidad de aprendizajes y ecologías ${ }^{1}$. Esta actividad de evaluación, buscaba una integración de los aprendizajes y revalorizar aquellos que acontecen en contextos informales predominantemente, o que hibridan con otras instancias o instituciones de formación, como es el caso de la docencia en educación superior.

En este sentido, nos enmarcamos en los planteos de Bruner (1997), quien destaca la importancia de las narrativas en educación para la creación de un mundo que dé significado a nuestras vidas, actos y relaciones. Por eso, con las distintas actividades de 
Edição Especial: I SSAPEC - Simpósio Sul-Americano de Pesquisa em Ensino de Ciências

ISSN: 2595- $4520 \quad$ Vol. 4, n. 3. 2021

aprendizaje nos enfocamos a fomentar un escenario en el que los docentes, a través de sus relatos, se conviertan en participantes activos y co-constructores de la cultura.

A los fines de este escrito, se analizaron las interacciones y los relatos desarrollados en el marco de un foro del aula virtual, en donde se compartió una consigna que les solicitaba pensar cómo aprenden Biología en su vida cotidiana. Para dicho análisis se consideraron las expresiones y vivencias proporcionadas por los docentes.

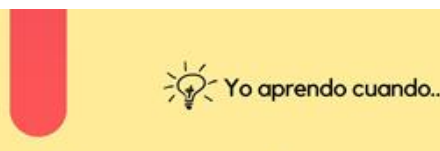

\section{¿Cómo aprendes Biologia/Geologia/ Ingenieria en tu vida cotidiana?}

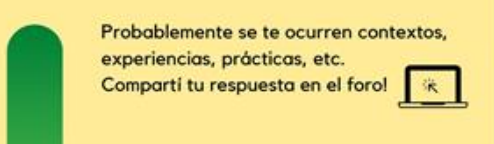

Figura 1 - Consigna de la actividad desarrollada mediante un foro de la plataforma virtual del curso y ejemplos de intervenciones de los distintos participantes.

\section{RESULTADOS}

Los análisis de los resultados mostraron que los profesores expresan en sus relatos una amplitud de vivencias, explicitando sus emociones en cuanto a los aprendizajes cotidianos, que muchas veces no son considerados como valiosos. Así, la tarea propuesta en el foro brindó una oportunidad para que los docentes reflexionen sobre sus roles en educación, haciendo explícito sus formas de aprender, a modo de conocer cómo los estudiantes aprenden en sus contextos cotidianos. Además, las interacciones generadas los llevaron a reflexionar acerca de la importancia de atender a las actualizaciones desde el campo pedagógico y de participar en instancias de formación donde temas como los contextos de aprendizaje, ecologías de aprendizaje y aspectos afectivos y emocionales sean retomados, conocidos y trabajados, desde perspectivas teóricas y desde la práctica. 
A partir de la revisión de la totalidad de las intervenciones en el foro, podemos decir que los participantes identifican una variedad de contextos cotidianos en los cuales aprenden cuestiones relativas a la Biología o a otras áreas de las Ciencias.

Se destacan en sus expresiones la observación directa de la naturaleza y fenómenos relacionados a ellas (rocas, paisajes, plantas, animales); la visualización de videos (series, documentales, películas); la búsqueda y lectura de materiales (búsqueda en internet, libros, artículos); diversas experiencias vividas en la vida cotidiana (paseos por el barrio; ocuparse del jardín; viajes) y en el trabajo (conversaciones con colegas y compañeros, salidas de campo); y el diálogo y la interacción con otras personas (familiares, docentes y amigos).

En este sentido, Palombo (2020) hace una descripción de distintos escenarios promisorios para el aprendizaje de la Biología, proponiendo y diversificándolos en siete formas y tipos: (1) los museos y centros de ciencias, (2) zoológicos, jardines botánicos y acuarios, (3) áreas naturales, (4) huertas y viveros, (5) activismos, movimientos y organizaciones sociales, (6) medios de comunicación, TIC y ciencia ciudadana y (7) la familia, hogar y vida cotidiana. En este marco, es preciso remarcar que en los relatos de los participantes, priman el hogar, los jardines y huertas y los medios y tecnologías de la información.

En cuanto a los aspectos emocionales las respuestas acentuaban aspectos positivos, manifestando curiosidad y agrado ante prácticas compartidas y el disfrute de ciertas actividades de aprendizaje verde. Así, a partir de las expresiones recabadas puede notarse que los aprendizajes construidos en diversos contextos cotidianos promovieron placer y satisfacción, lo que contribuye al bienestar subjetivo.

A continuación compartimos algunos fragmentos de respuestas proporcionadas a la tarea. En primer lugar, se destacan expresiones referidas a experiencias cotidianas en donde los participantes reconocen oportunidades para aprender:

Aprendo biología un poco amarillista en los documentales que veo con mucha frecuencia y a veces en el campo durante mi trabajo, por ejemplo ayer en el norte cordobés ví una corzuela y cuando llegué a casa me puse a googlear sobre el animal. (Participante $\left.N^{o} 1\right)$

Biología aprendo de muchas formas, yendo a las sierras, con mis binoculares, pescando, etc. o sea, observando. También probando, cambiando las plantas de lugar de mi casa a ver si crecen mejor o cambiando el filtro del estanque de la casa de mis viejos. Hay un proceso 
que se itera, observo algo, si no me gusta lo cambio y veo si mejora, si no lo vuelvo a cambiar. (Participante $N^{o} 3$ )

Estos fragmentos ponen de relieve la importancia que le atribuyen los participantes a la observación y el contacto directo con la naturaleza en el desarrollo de sus aprendizajes, emergiendo también aspectos como la curiosidad y el disfrute por aprender.

Los siguientes extractos muestran otras expresiones pero que siguen la misma línea; observación, prueba y curiosidad continúan siendo consideradas como importantes:

Aprendo Biología cuando me duele algo porque necesito saber qué hay dónde me duele. Pero también aprendo al intentar cuidar mis cítricos y observando y probando con la huerta. Además hace años que en casa hacemos compost, así que también aprendo esos ciclos observando y haciendo. En todos los casos, veo que hay un disparador que es la curiosidad, observo, tomo nota, busco en internet y luego hago una conclusión. (Participante $N^{o} 4$ )

En cuanto a Biología creo que la forma en que aprendo día a día es con las plantas que intento mantener vivas o con los animales que tengo en casa y que no son pocos. También un poco mirando documentales o programas del estilo. En el campo he aprendido por curiosidad. La Patagonia ofrece una flora y una fauna tan distinta a la de Córdoba que obliga al que observa intentar aprender. (Participante $N^{\circ} 5$ )

En el fragmento que compartimos a continuación, la participante enumera una serie de contextos o formas que aprende informalmente, retomando así algunas cuestiones ya mencionadas pero también agregando otras:

Contextos de aprendizaje de Biología, Ingeniería y Geología, en la vida cotidiana, se me ocurren muchos:

*Diálogos con otras personas (familiares, amigos, colegas)

*Radio (cuando se habla de algún tema relacionado con estas ramas del conocimiento)

*Propagandas en la televisión, documentales, películas y series relacionadas a estas temáticas

*Internet (consultas que uno hace para aprender a hacer algo, como el compost, una huerta, arreglar algún electrodoméstico en casa)

*Cuando visitamos museos, zoológicos, acuarios, parques nacionales, en excursiones guiadas

*Cuando hay que arreglar algo en casa, o hacer algo que implique prueba y error

* En las actividades diarias de jardinería

*Cuando salimos a pasear, o a andar en bicicleta, o cualquier actividad que implique un contacto con la naturaleza

*En las consultas que uno hace con distintos médicos, o con el veterinario cuando ve a sus mascotas.

Creo que todos los días aprendemos algo nuevo en nuestras actividades cotidianas. (Participante $N^{o}$ 6) 
Por último, compartimos otros dos fragmentos en donde los participantes reconocen cómo aprenden en diversos contextos cotidianos, destacando también la relevancia de la observación, la interacción continua con el medio y el papel de publicaciones y de diversas producciones audiovisuales a las que se puede acceder a partir de las TIC.

Hola, yo aprendo biología en mi vida cuando salgo a avistar aves en mis tiempos libres, cuando leo publicaciones relacionadas a la ecología, entomología o taxonomía. Cuando veo documentales acerca de conservación o de relación presa-predador, etc. Aprendo geología en los momentos en que busco fósiles o analizo las piedras que colecto porque me resultaron interesantes. En cualquier caso mis formas de aprendizaje son interactuando con mi entorno, dejando que mi imaginación y curiosidad sean quienes guíen la adquisición de conocimientos. (Participante $N^{o} 10$ )

Hola! Creo que mis aprendizajes de estas disciplinas en la vida cotidiana, son casi continuos. Sigo aprendiendo de geología cuando veo paisajes, cuando escucho noticias relacionadas a fenómenos (generalmente desastres) naturales, cuando converso con gente acerca de sus contacto cotidiano con los recursos naturales (suelos, aguas, minerales, etc.), cuando miro series y documentales, cuando visito museos y hasta cuando leo novelas y cuentos. La base científica que he adquirido durante el cursado de mi carrera, me permite formalizar estos conocimientos y acomodarlos dentro del marco conceptual que he ido construyendo. Respecto a la biología y la ingeniería, la forma de aprender es bastante similar a la mencionada para temas geológicos, añadiendo también actividades más prácticas, como pueden ser arreglar algún elemento electrónico o intentar conseguir que no se mueran las plantas del balcón. Sin embargo, al no tener mucho conocimiento de las bases de estas disciplinas, quizás los aprendizajes en estas temáticas son un poco más superficiales. (Participante $N^{\circ} 13$ )

Como puede notarse en los fragmentos compartidos, son muy variados los contextos cotidianos en los que los participantes reconocen aprender cuestiones relacionadas con las Ciencias y que son valorados muy positivamente. Así, la tarea diseñada en el curso fue un disparador para pensar estas dos cuestiones: los contextos en que se aprende y las emociones asociadas con ellos. Las reflexiones finales, que exponemos a continuación, se orientan en este mismo sentido: a resaltar la importancia de tareas como la aquí comentada.

\section{CONSIDERACIONES FINALES}

A partir del trabajo presentado, podemos realizar a modo de cierre algunas consideraciones. Nos parece interesante reflexionar principalmente sobre dos puntos: por un lado, acerca de la importancia de incluir en la formación docente, propuestas y temas novedosos y que promuevan emociones beneficiosas para los aprendizajes; y por Recebido em: 18/01/2021

Aceito em: 24/02/2021 
Edição Especial: I SSAPEC - Simpósio Sul-Americano de Pesquisa em Ensino de Ciências ISSN: 2595- $4520 \quad$ Vol. 4, n. 3. 2021

otro lado, sobre la relevancia que tiene para la formación de profesores en ciencias, conocer otros tipos de ámbitos en los cuales pueden intervenir y fomentar el desarrollo de actitudes acordes a estos nuevos desafíos.

Creemos que resulta difícil identificar qué, cómo y por qué las personas participan en actividades de aprendizaje informal; ya que el aprendizaje informal ocurre todo el tiempo y en todas partes para todos, por ello, es necesario observar e investigar continuamente sobre el fenómeno (MIYAKE, 2017); generando de este modo, en el área de la biología, posibilidades para la co-construcción de saberes y conocimientos de la vida cotidiana y la apertura, en el caso de la universidad, a experiencias de docencia, investigación y extensión.

La tarea aquí comentada, propuesta en el marco de un curso de formación docente, tuvo entre sus principales objetivos movilizar a los docentes y proporcionar oportunidades para que puedan reflexionar acerca de la riqueza de los propios aprendizajes construidos fuera del ámbito escolar. Consideramos que la actividad cumplió con el objetivo mencionado generando una ocasión para la reflexión en donde, a partir de reconocer los diversos ámbitos y contextos en que el propio profesor aprende, pueda luego generar propuestas en donde se hibriden estos saberes y conocimientos y pueda ofrecer a los estudiantes variados escenarios y situaciones de aprendizaje. Ello no sólo con el propósito de generar propuestas más enriquecedoras y más amplias, sino también apuntando a que estas propuestas promuevan emociones beneficiosas para los aprendizajes y en donde esas emociones sean reconocidas. Para ello, sería necesario que se creen las condiciones para que los estudiantes puedan pensar y reflexionar sobre aquello que sienten al momento de aprender, pudiendo identificar qué actividades, tareas, situaciones o contextos, suscitan emociones positivas tales como el disfrute, la pasión, la esperanza o el orgullo ante los aprendizajes construidos.

Creemos que actividades de este tipo contribuyen a repensar los contextos de aprendizajes y a reconocer su importancia y así, tal vez, alentamos a los docentes a que en sus propias clases y propuestas educativas abran las puertas a las experiencias cotidianas de los estudiantes. Consideramos que es necesario y relevante reconocer que experiencias tales como visitar un museo o un acuario, realizar un viaje, observar y tomar contacto con la biodiversidad que nos rodea, buscar información en internet, ver series o documentales pueden convertirse en experiencias que despierten la pasión y la 
curiosidad (PALOMBO, 2020; REISS; McCOMAS, 2020). Así, atender a ello brinda aportes orientados al diseño de propuestas educativas más significativas para los estudiantes, que sean valoradas intrínsecamente por ellos y en las que, al involucrarse en ellas -tanto docentes como estudiantes- experimenten emociones como el placer, la alegría y el disfrute por aprender.

Siguiendo esta línea, y en acuerdo con lo expresado por García Romano y Martín (2020), acentuamos la idea de que investigar e incorporar innovaciones pedagógicas que contemplen estos temas (i.e. saberes cotidianos, contextos de aprendizaje y emociones y sus relaciones) invita a pensar en alternativas promisorias en la formación de los profesionales de la educación. Es decir, frente a un panorama heterogéneo y dinámico marcado por la complejización y la extensión de los contextos educativos, los profesores ya no solo deberían ajustar sus interacciones en espacios formales, sino más bien, inevitablemente, ser conocedores, conectores e interventores entre diversas situaciones de aprendizaje. Estos aportes se orientan a la formación de un profesorado que sea capaz de incluir nuevas concepciones sobre el aprendizaje, diferentes formas de intervenir en el aula y más allá de ella y que, principalmente, pueda desarrollar actitudes que contribuyan a asumir éticamente las responsabilidades que implican estos nuevos ambientes de intervención (GARCIA ROMANO; MARTÍN, 2020).

En la formación del profesorado es importante revalorizar aquellos sitios formales-informales (REISS; McCOMAS, 2020), hibridando estos diversos contextos de aprendizaje -formal e informal-, creando y co-construyendo nuevas ecologías que permitan combinar ámbitos, comunidades y personas. En la consideración de la diversidad de los aprendizajes, es preciso resaltar las apreciaciones de Rosa y Paula (2020) acerca de la importancia de considerar en los procesos de formación docente, un itinerario con diálogos fructíferos con y en los territorios, específicamente con los saberes populares, ancestrales y tradicionales que los estudiantes co-construyen culturalmente.

Creemos que en la formación profesional es importante hibridar diversos contextos y fomentar el abordaje de líneas y metodologías de investigación, basadas en el reconocimiento de la pluralidad de conocimientos y saberes heterogéneos, y en las 
Edição Especial: I SSAPEC - Simpósio Sul-Americano de Pesquisa em Ensino de Ciências

ISSN: 2595- $4520 \quad$ Vol. 4, n. 3. 2021

interconexiones continuas y dinámicas entre ellos, pensando en un interconocimiento, formulado y mirado desde una perspectiva intercultural (DE SOUSA SANTOS, 2010).

\section{NOTAS}

${ }^{1}$ Una de las lecturas sugeridas durante el curso fue la autobiografía de Darwin, tomando como base planteos de Reiss y McComas (2020), quienes destacan la importancia de aprender sobre la naturaleza de la ciencia desde narraciones escritas, tomando como ejemplo escritos de Darwin y una visita a una propiedad histórica.

${ }^{2}$ La corzuela parda (Mazama gouazoubina) es un ungulado sudamericano, cuya distribución alcanza el norte y centro de Argentina.

\section{AGRADECIMIENTOS}

Los autores agradecen a la Mg. Gabriela Giammarini por la revisión gramatical y a quienes asistieron al curso de formación por la participación en las diversas actividades propuestas durante su desarrollo.

\section{REFERENCIAS}

ANDERSON, David y ELLENBOGEN, Kirsten M. Learning science in informal contexts-epistemological perspectives and paradigms. En FRASER, B. J. et al. (Eds.). Second International Handbook of Science Education 24. New York: Springer Science and Business Media B.V., 2012. p. 1179-1187.

BRUNER, Jerome. La fábrica de historias: derecho, literatura y vida. Buenos Aires: Fondo de Cultura Económica Argentina, 2013.

BRUNER, Jerome. La educación, puerta de la cultura. Barcelona: Aprendizaje Visor, 1997.

DARWIN, Charles. Autobiografía Charles Darwin. España: Omegalfa. (s/f). p. 71.

DE SOUSA SANTOS, Boaventura. Descolonizar el saber, reinventar el poder. Montevideo: Editorial Trilce. 2010. 
Edição Especial: I SSAPEC - Simpósio Sul-Americano de Pesquisa em Ensino de Ciências

ISSN: 2595- $4520 \quad$ Vol. 4, n. 3. 2021

ESHACH, Haim. Bridging in-school and out-of-school learning: Formal, non-formal, and informal education. Journal of science education and technology, v. 16, n. 2, 171190, 2007.

FALK, John H. Free-choice environmental learning: Framing the discussion. Environmental Education Research, v. 11. n. 3, 265-280, 2006.

FORESTO, Emiliano. Aprendizajes formales, no formales e informales. Una revisión teórica holística. Contextos de Educación, n. 29, 24-36, 2020. Disponible en: http://www2.hum.unrc.edu.ar/ojs/index.php/contextos/article/view/1142 Acceso en: 14 dic. 2020.

FRIED, Leanne, MANSFIELD, Caroline y DOBOZI, Eva. Teacher emotion research: Introducing a conceptual model to guide future research. Issues in Educational Research, 25(4), 415-441, 2015. Disponible en: http://www.iier.org.au/iier25/fried.pdf Acceso en: 10 dic. 2020.

GARCÍA ROMANO, Leticia y MARTÍN, Rocío Belén. Hacia una nueva ecología en investigación educativa en ciencias biológicas. Boletín Biológica, n. 44, 4-9, 2020.

KIM, Mijung y DOPICO, Eduardo. Science education through informal education. Cultural studies of science education, v. 11, n. 2, 439-445, 2016.

MARTÍN, Rocío Belén. Perspectiva teórica sobre el estudio de los contextos y comunidades de aprendizaje. En MARTÍN, R., PAOLONI, P. V. y M.C. RINAUDO (comps.) Comunidades. Estudios y experiencias sobre contextos y comunidades de aprendizaje. Villa María, Córdoba: Eduvim. 2019. p. 75-131.

MARTÍN, Rocío Belén y DONOLO, Danilo Silvio. Aprendizajes informales. Perspectivas teóricas y relatos de aprendizajes. Ikastorratza, e-Revista de didáctica, n.23, p. 115-131, 2019. DOI: 10.37261/23_alea/5

MIYAKE, Shiho. Learning Science in Informal Contexts. En TABER K.S.; AKPAN B. (eds.) Science Education. New Directions in Mathematics and Science Education. Rotterdam: SensePublishers, 2017. p. 430-442. Disponible en: https://doi.org/10.1007/978-94-6300-749-8_3 Acceso en: 10 set. 2020.

PALOMBO, Nahuel. Aprender Biología en contextos diversos. En MARTÍN, Rocío; PALOMBO, Nahuel; MANAVELlA, Agustina; VAJA, Arabela; DÍAZ LOZADA, 
Edição Especial: I SSAPEC - Simpósio Sul-Americano de Pesquisa em Ensino de Ciências

ISSN: 2595- $4520 \quad$ Vol. 4, n. 3. 2021

José; GARCIA, Leticia. Experiencias y aprendizajes en clave autobiográfica. Aportes teóricos y prácticos sobre contextos, compromiso y emociones en la formación docente. Córdoba: Universidad Nacional de Córdoba. 2020, en prensa.

PEKRUN, Reinhard. The Control-Value Theory of achievement emotions: assumptions, corollaries, and implications for Educational Research and practice. Educational Psychology Review, 18, 315-341, 2006.

PEKRUN, Reinhard. Emotions and Learning. Educational Practices 24. International Academy Education and International Bureau of education. 1-32, 2014. Disponible en:

http://www.ibe.unesco.org/fileadmin/user_upload/Publications/Educational_Practices/E dPractices_24eng.pdf Acceso en: 10 set. 2020.

REISS, M. J. y McCOMAS, W. F. Informal Learning Sites and Their Role in Communicating the Nature of Science. En McCOMAS, William (Ed.). Nature of Science in Science Instruction, pp. 711-729. Cham: Springer. 2020.

RENNIE, Léonie J. Learning science outside of school. En LEDERMAN, Norman; ABELL, Sandra (Eds.), Handbook of Research on Science Education Volume II, pp. 120-144. USA: Routledge. 2014.

ROSA, Marina Comerlatto da.; PAULA, Adalberto Penha da. Diálogos entre Educação do Campo e Ensino de Ciências: possibilidades na formação de professoras/es de ciências da natureza. Revista Insignare Scientia - RIS, v. 3, n. 4, 3-21, 20 nov. 2020.

VAJA, Arabela. La importancia de los relatos en los contextos educativos. Reflexiones desde los aportes de Bruner. IKASTORRATZA. e-Revista de Didáctica, n.12, 1-9, 2014. Disponible en: http://www.ehu.eus/ikastorratza/12_alea/Bruner.pdf Acceso en: 14 dic. 2020. 